\title{
Developing Sustainable Trading Strategies Using Directional Changes with High Frequency Data
}

\author{
Ailun Ye \\ Business School, University of Greenwich,London, UK \\ alanye0412@qq.com \\ Antoaneta Serguieva \\ Computational Intelligence Research Group, \\ University of Portsmouth, Portsmouth, UK \\ and CCFEA, University of Essex, Colchester
}

\author{
V L Raju Chinthalapati \\ Business School, University of Greenwich, London, UK \\ v.1.r.chinthalapati@greenwich.ac.uk \\ Edward Tsang \\ Centre for Computational Finance and Economic Agents CCFEA \\ University of Essex, Colchester, UK \\ edward@essex.ac.uk
}

\begin{abstract}
Market prices are traditionally recorded in fixed time intervals. Directional Change is an alternative approach to summarize price movements in financial markets that is consistent with across all time scales. Unlike time series, directional change summarizes the big data in finance by focusing on the intrinsic time of the data. This captures deeper intrinsic data qualities and thus trading strategies based on directional change are more sustainable and less disruptive. In this paper, we propose four trading strategies using the concept of directional change and explore the combination with technical analysis. The trading strategies are tested using EUR/USD and GBP/USD high frequency FX market data. Empirical results show good performance of our trading strategies based on thresholds, and that combining with technical analysis brings further improvement.
\end{abstract}

Keywords- FX trading; directional changes; sustainable trading strategies.

\section{INTRODUCTION}

In information economy, advanced technology and innovation in financial markets and Multilateral Trading Facilities (MTFs) made the financial data has become abundant.Availability of high frequency data opens the doors for more information regarding the market microstructure. The success of an investor in the market depends on how well the investor is analysing the available data and predicting the future movements of the market. Traditionally, time-series analysis is used to analyse and model the given financial data. Time-series analysis concerns the analysis of the available data in order to extract the underlying structure and patterns in the data. For that, the changes in a financial variable (for example, price) are modelled as a random process and the dynamics involved is formulated as stochastic difference equations in order to extract the internal structure (or the time varying properties) of the underlying data. The parameters of the model are calibrated by using the financial data so that the model is used for predicting the future changes in the financial variable. In traditional time-series analysis, we consider extrinsic time (or physical time), where the flow of time is independent from the changes that occur in a given financial variable. That means, time is considered as an absolute entity, where the time ticks are independent of the events in the market. One of the challenges in time-series analysis is that the models are unable to characterize the nature of the price changes consistently across all time intervals. For example, a time-series model that explains the changes in the financial variable at low frequency time intervals need not be successful at high frequency time intervals. For the time-series analysis, the financial data is collected at regular time intervals. But it is well known fact that the trading events (or price changes) are a sequence of irregularly time-distributed events and they need not be occurring at uniform time intervals. One of the drawbacks with the regular interval timeseries is that there is no guarantee that the events (or price changes) in the market would fall precisely on the regularly spaced time ticks of the physical time and the ignored details in between the time ticks are not significant enough.

Reference [7] projects directional change (DC) as a new approach to summarize the markets' prices movement. Unlike time series, the DC framework focus on the intrinsic time of market movement rather than physical time. The DC framework has been proved helpful in summarizing the financial market. Reference [8] propose 12 independent scaling laws by analyzing 13 different currency exchange rates using the DC concept. Reference [9] introduce the Scale of Market Quakes to quantifying FX market activity on the DC concept. Reference [10] introduce indicators and provide an approach to profiling equities on the UK stock market using DC-based analysis. The DC framework throws light on the consistent patterns in the financial data that are sustainable at all time scales that are useful in developing more reliable and sustainable trading strategies.

The literature on trading strategies contains plenty of trading models.Some trading models are based on the stylized facts of a market (e.g. [1]). Other trading strategies using technical trading rules (e.g. [2-3]). Momentum models are also one approach (e.g. [4-5]). The literature also includes multiple trading strategies (e.g. [6]). The criterion common to all these 
researches is using time series analysis, which considers physical time intervals.

Recently, high frequency traders develop trading models based on the DC concept. Reference [11] introduce a trading strategy that exploit the scaling laws in FX market. Reference [12] present a trading strategy based on the daily closing price by combining the DC concept and Genetic Programming. More recently, [13] propose a contrarian trading strategy based on the DC concept and test it using two currency pairs.

We believe that applying the DC framework to develop a trading strategy has not been fully exploited. In this paper, we propose four trading models using the DC concept and test them in FX markets. We also explore the possibility to combine the DC concept with the technical analysis. We verify that our strategy is profitable at certain thresholds. The experiments are conducted using two currency pairs: EUR/USD and GBP/USD.

This paper continues as follow: Section II describes the general framework of the DC concept and section III introduce the four trading strategies. The experiments are provided at section IV. Report and discussion of the results are in section $\mathrm{V}$. In the end, we conclude in section VI.

\section{DIRECTIONAL CHANGE}

Scaling law is a law that describes the scale invariance found in many natural phenomena[14]. In finance, there is one law first sparked in 1990 that has been widely reported. It shows the scaling relations in FX data that the mean absolute change of logarithmic mid-prices is scale-invariant to the time interval of its occurrence[15].

Later in 1997, reference [7] propose a scaling law about the price change of a threshold, which is so-called directional changes.

$$
\mathrm{N}\left(\Delta_{X d c}\right)=\left(\frac{\Delta_{X d c}}{c_{N, d c}}\right)^{E_{N, d c}}
$$

Where $\mathrm{N}\left(\Delta_{X d c}\right)$ is the number of directional changes, $\Delta_{X d c}$ is the DC sizes. The DC approach focuses on significant changes in price movements. The change is defined as a certain percentage price move. Let $\theta$ be the threshold, which is the percentage change. According to the DC concept, the market can be one of the two forms: downturn event or upturn event. A downturn event is directly followed by an upturn event; and vice versa. A downturn event consists of a downturn DC and a downturn overshoot. Similarly, an upturn event consists of an upturn DC and upturn overshoot. If we observe a price rise of magnitude $\theta$, we say the market is in upturn DC; vice versa. Let $p_{c}$ be the current price of the market and $p_{\text {ext }}$ be the price where a downturn or upturn event begins. In the case of a downturn event, the $p_{\text {ext }}$ is the highest price of the downturn DC while in the case of an upturn event, the $p_{\text {ext }}$ is the lowest price of the upturn DC. A DC event is confirmed when we observe the price change in $p_{c}$ satisfies (2).

$$
\left|p_{c}-p_{\text {ext }}\right| \geq \theta * p_{\text {ext }}
$$

If (2) holds, the time where $p_{\text {ext }}$ is observed is called an extreme point and the time where $p_{c}$ satisfied is called a DC confirmation point. The price movement between the extreme point and the DC confirmation point form the DC event. In the case of a downturn event, the downturn DC is observed whenthe $p_{c}$ satisfies (2). Then we start to look for the next upturn DC. The new $p_{\text {ext }}$ will be highest price after the previous downturn DC. When the upturn DC is observed, the new $p_{\text {ext }}$ is the extreme point of the upturn DC. The interval between the downturn confirmation point and the new upturn extreme point is called Overshoot (OS). In this case, the overshoot event is a downturn overshoot located between a downturn DC and an upturn DC.

\section{STRATEGY}

Reference [8] enlarged the catalogue of FX stylized facts by observing 12 new empirical scaling laws that hold for close to three orders of magnitude and cross 13 currency exchange rates. It verified that the coastline, the sum of all price moves of a given threshold, measures on average $6.4 \%$ per day at $0.05 \%$ threshold, which is astonishingly long. In this section, we introduce four new trading strategies based on the DC concept and scaling law. The strategy 1 and 2 shared the same algorithm at the entry point but different ways sending order. Strategy 1 use fixed order while strategy 2 use trailing stop. Strategy 3 use a different algorithm to close order. Strategy 4 use similar algorithm as strategy 3 has, but combined used with Directional Movement Index (DMI). We will describe the difference between each strategy.

\section{A. Strategy 1: Fixed Target DC}

In the DC concept, a downturn DC is always followed by a downturn overshoot (OS) and an upturn DC is always followed by an upturn DC. The period and the length of the OS is not known. It is scaled as extension periods of the corresponding DC events in the minor trend of a full DC event. It is believed that there is highly possibility that the trend will continue after a DC event is confirmed. The strategy 1 exploits the idea.

Strategy 1 opens a long position at the confirmation point of an upturn DC and believes it will further be followed by an upturn OS. The order is sent as a fixed target order with stop loss level and take profit level. Once the order reaches either stop loss or take profit, it will be fulfilled and close the position. The algorithm could be described as follow:

If (Current trend is upturn) and $\left|p_{c}-p_{\text {ext }}\right| \geq \theta * p_{\text {ext }}$ Open position: Buy Order, StopLoss, TakeProfit;

\section{B. Strategy 2: Trailing Stop DC}

In strategy 2, we build similar algorithm as strategy 1 has. The order in strategy 1 is fixed order, where the stop loss and take profit level is fixed figure when sent to the market. We try 
to explore the possibility that the trailing stop as a correction of fix order will increase the profitability of the strategy.

Different from fixed order, trailing stop does not have a fix level of exit. In setting up the trailing stop, we first send an order to the market with an initial stop loss. The order could either be stopped at this stop loss level or make certain pre-set profit to trigger trailing loss system. After some amount of profit is made, the new trailing stop loss is activated and updates the old stop loss level. The trailing stop loss line is the stop loss from the current maximum profit instead from the entry price. It ensures the risk is controlled at the given trailing level, but it is still able to catch more profits rather than end position at fixed take profit target. The system could be described as follow:

If (Current trend is upturn) and $\left|p_{c}-p_{\text {ext }}\right| \geq \theta * p_{\text {ext }}$ Open position: Buy Order, Initial StopLoss, Initial TakeProfit If OrderStatus = HasNotFilled, then

If Position.Profit > MaxProfit MaxProfit $=$ Position. Profit;

If Position.Profit >Initial TakeProfit Update Position.StopLoss = MaxProfit TrailingStopLoss;

\section{Strategy 3: Close Threshold DC}

Strategy 3 is also based on directional change events. Compared to the previous 2 strategies, strategy 3 has the same entry point where the directional change events are confirmed. But it has a different exit timing. According to the scaling laws of intrinsic time framework, an overshoot event usually has the same width of a directional change event, which means it usually has a $\theta$ percentage price movement over the direction. Strategy 3 tries to catch this overshoot trend and close position before the trend turns into downward direction. By defining a close threshold, it may close earlier than the downturn point. This close threshold is defined as follow:

$$
\begin{aligned}
\text { closeThreshold } & =k * \text { Threshold } * e^{-O S V} \\
O S V & =\frac{P_{D C C}-P_{\text {ext }}}{P_{D C C} * \theta}
\end{aligned}
$$

Where $\mathrm{k}$ is a parameter, threshold is given by the trader, OSV is the overshoot value calculated by the formula, $P_{D C C}$ is the price at the directional change event confirmed. When OSV value equals to 1 , it means the price has moved same $\theta$ percentage change into the direction, which is the expectation movement in statistics.

The strategy algorithm could be described as follow:
If $P_{c} \geq P_{\text {ext }} *(1+$ closeThreshold $)$ where closeThreshold $=k *$ Threshold $* e^{-O S V}$, OSV $=\frac{P_{D C C}-P_{\text {ext }}}{P_{D C C} * \theta}$ Close the long position;

\section{Strategy 4: DMI Trend DC}

The fourth strategy aimed to make a combination of directional change and technical analysis, hoping it will have each's advantage. The strategy 3 is a long only strategy. Therefore, if strategy 3 could run in a steady uptrend of the market, it will have a better win rate. Strategy 4 exploit this idea with a technical indicator.

There are many technical indicators in defining trend. Here we use the Directional Movement Index, which is also known as DMI, developed by Welles Wilder. DMI shows the strength of a trend either up or down. The DMI is composed by three indicators: ADX, +DI and -DI. The Average Directional Index (ADX) line shows how strong the trend is. The higher it is, the stronger the trend. According to Wilder, a trend is present when it is greater than 25 . When it falls below 25 , there is no trend. The Positive Direction Indicator (+DI) and Negative Direction Indicator (-DI) show the current price movement. When +DI is above -DI, it is an upward trend. When -DI is below $+\mathrm{DI}$, it is a downward trend.

The idea for strategy 4 is to run the strategy 3 only when DMI shows the market is in an upward trend. The strategy 4 is as follow:

If (Current DC trend is upturn) and $\left|p_{c}-p_{\text {ext }}\right| \geq \theta * p_{\text {ext }}$ and (Current DMI trend is upward)

Open position: Buy Order;

If $P_{c} \geq P_{\text {ext }} *(1+$ closeThreshold $)$ where

closeThreshold $=k *$ Threshold $* e^{-O S V}$, OSV $=\frac{P_{D C C}-P_{\text {ext }}}{P_{D C C} * \theta}$

Close the long position;

TABLE I. STRATEGY SUMMARY

\begin{tabular}{|l|l|l|}
\hline \multicolumn{1}{|c|}{ Strategy } & \multicolumn{1}{|c|}{ Name } & \multicolumn{1}{c|}{ Note } \\
\hline 1 & Fixed Target DC & $\begin{array}{l}\text { Fixed target order at } \\
\text { confirmation points }\end{array}$ \\
\hline 2 & Trailing Stop DC & $\begin{array}{l}\text { Trailing stop order at } \\
\text { confirmation points }\end{array}$ \\
\hline 3 & Close Threshold DC & $\begin{array}{l}\text { Open at confirmation } \\
\text { points, close through Close } \\
\text { Threshold }\end{array}$ \\
\hline 4 & DMI Trend DC & $\begin{array}{l}\text { Combination of DMI and } \\
\text { DC, only run Strategy 3 } \\
\text { when DMI shows an } \\
\text { uptrend }\end{array}$ \\
\hline
\end{tabular}




\section{EXPERIMENTS}

We test our proposed trading strategy in the foreign exchange markets using two most popular pairs EUR/USD and GBP/USD. In Section A, we describe how we prepare the data for the experiments. In Section B, we list the evaluation metrics used to assess the strategy performance.

\section{A. Prepare the Dataset}

The foreign exchange opens five days a week, excluding weekend. The data continually started from Monday 00:00 to Friday 22:00. The data sample covers the period from 1st Jan. 2015to 30st Jun. 2016. The original data set contains millions of real ticks trading including bid price and ask price. The data is divided into two parts for in-sample testing and out-ofsample testing. The first year data from 01/01/2015 to $31 / 12 / 2015$ is used for back testing. It runs the sample data as a history data back testing to generate the best possible variables for each parameter. Then we apply all the parameters with best variables into the second half data which is from 01/01/2016 to $30 / 06 / 2016$. This part testing is the demo testing which tries to simulate a real run through the out-of-sample data.

The strategies are back tested in high frequency trading data which is based on ticks. Results in back testing part are only used for identifying best parameters. The other part of data is used as out of sample period data. The results in demo testing are more convincible because it won't forerun the data and it simulates a real trading environment.

It is assumed that there is no commission charged in the experiments. Order is instantly sent and filled without slippage. The long trade is executed at the price of ask at the moment it is sent and the short trade is executed at the price of bid. The testing starts with an account at balance 100,000 dollars. Each order has same size 100,000 contracts which is 1 lot. Ten thresholds from $0.01 \%$ to $0.9 \%$ will run for each strategy

TABLE II. PART OF THE DATA

\begin{tabular}{|c|c|c|c|}
\hline Instrument & Time & Bid & Ask \\
\hline EUR/USD & $\begin{array}{l}20160104 \\
00: 00: 00.057\end{array}$ & 1.08516 & 1.08522 \\
\hline EUR/USD & $\begin{array}{l}20160104 \\
00: 00: 00.719\end{array}$ & 1.08516 & 1.0852 \\
\hline EUR/USD & $\begin{array}{l}20160104 \\
00: 00: 00.873\end{array}$ & 1.08515 & 1.0852 \\
\hline EUR/USD & $\begin{array}{l}20160104 \\
00: 00: 01.064\end{array}$ & 1.08518 & 1.0852 \\
\hline EUR/USD & $\begin{array}{l}20160104 \\
00: 00: 01.184\end{array}$ & 1.08518 & 1.08523 \\
\hline EUR/USD & $\begin{array}{l}20160104 \\
00: 00: 01.196\end{array}$ & 1.08519 & 1.08524 \\
\hline EUR/USD & $\begin{array}{l}\text { 20160104 } \\
\text { 00:00:01.217 }\end{array}$ & 1.08519 & 1.08523 \\
\hline EUR/USD & $\begin{array}{l}20160104 \\
00: 00: 01.228\end{array}$ & 1.08521 & 1.08523 \\
\hline EUR/USD & $\begin{array}{l}20160104 \\
00: 00: 01.237\end{array}$ & 1.08521 & 1.08526 \\
\hline EUR/USD & $\begin{array}{l}20160104 \\
00: 00: 01.249\end{array}$ & 1.08522 & 1.08526 \\
\hline
\end{tabular}


case happens at threshold $0.01 \%$ where EUR/USD drops nearly $25 \%$ and GBP/USD fails all the balance. As the threshold increases, the total trades (table IX) and the balance drawdown decrease (table V). One could make this strategy into a portfolio with different thresholds to diverse drawdown risk and adjust the frequency of trades. The winning rate and profit factors do not have clear correlation with thresholds but still a good indicator to check the performance of this threshold. In all, strategy 1 is a very profitable strategy in EUR/USD and adjustable with risk and frequency by changing threshold.

TABLE III. TOTAL PROFIT OF EUR/USD

\begin{tabular}{|l|l|l|l|l|}
\hline Threshold & Strategy 1 & \multicolumn{1}{|c|}{ Strategy 2 } & Strategy 3 & Strategy 4 \\
\hline $0.01 \%$ & $-24,973$ & $-13,016$ & 11,207 & $-3,102$ \\
\hline $0.03 \%$ & 61,493 & 97,687 & 21,319 & -98 \\
\hline $0.05 \%$ & 40,394 & 39,790 & 17,887 & 3,144 \\
\hline $0.07 \%$ & 62,449 & 62,707 & 13,413 & 1,450 \\
\hline $0.09 \%$ & 28,628 & 47,842 & 13,620 & -615 \\
\hline $0.1 \%$ & 48,543 & 48,126 & 5,874 & $-2,243$ \\
\hline $0.3 \%$ & 1,038 & 3,278 & 7,565 & -363 \\
\hline $0.5 \%$ & -300 & -218 & -299 & 196 \\
\hline $0.7 \%$ & $-1,599$ & $-1,477$ & -830 & 867 \\
\hline $0.9 \%$ & 1,100 & 2,174 & 4,396 & - \\
\hline
\end{tabular}

TABLE IV. TOTAL PROFIT OF GBP/USD

\begin{tabular}{|l|l|l|l|l|}
\hline Threshold & Strategy 1 & \multicolumn{1}{|c|}{ Strategy 2 } & Strategy 3 & Strategy 4 \\
\hline $0.01 \%$ & $-99,951$ & 55,470 & $-3,784$ & 5,879 \\
\hline $0.03 \%$ & $-51,936$ & 13,241 & 12,484 & 1,160 \\
\hline $0.05 \%$ & $-19,100$ & $-3,273$ & 2,640 & 3,122 \\
\hline $0.07 \%$ & $-13,440$ & 9,257 & 3,132 & 361 \\
\hline $0.09 \%$ & $-13,247$ & 2,567 & $-2,516$ & $-2,273$ \\
\hline $0.1 \%$ & $-4,737$ & 48,758 & 1,108 & -325 \\
\hline $0.3 \%$ & 2,384 & 5,793 & 8,232 & 2,997 \\
\hline $0.5 \%$ & 767 & -328 & $-14,372$ & 1,018 \\
\hline $0.7 \%$ & 652 & 1,732 & $-10,935$ & 372 \\
\hline $0.9 \%$ & -350 & 739 & $-14,993$ & $-2,052$ \\
\hline
\end{tabular}

Strategy 2 uses similar concept as strategy 1. They have same position entrance where the confirmation point of downturn directional change event is. The difference is strategy 2 continues to trade when strategy 1 closes at the profit taken level. Strategy 2 uses trailing stop which is a floating stop loss line to catch the potential profits in the future trend. It will close the order once it has particular pips lost from the maximum profit level, which is like a drawdown of the order. In the case of profitable maneuver, strategy 2 will catch more potential profits because of the trailing stop rather than exit at a precise take profit level. As expect, strategy 2 do produce more returns than strategy 1 . In table III EUR/USD, strategy 2 has an average return at $28.69 \%$ for all thresholds while strategy 1 has an average return at $21.68 \%$. There are also 7 out of 10 positive returns, but for each individual instance, strategy 2 has higher return than strategy 1 in most cases except in threshold $0.05 \%$ and $0.1 \%$ where the returns are $40.39 \%$ and $39.79 \%, 48.54 \%$ and $48.13 \%$, which is quite closed. In most cases in EUR/USD strategy 2 has a good correction in returns. For GBP/USD at table IV, strategy 1 generates negative return in most thresholds. But strategy 2 produces positive result in 8 out of 10 cases while only 3 out of
10 work in strategy 1 . It is a fail at threshold $0.01 \%$ in strategy 1 but in strategy 2 it gets $55.47 \%$ which is a very high number. At threshold $0.1 \%$, strategy 2 reaches another high return $48.76 \%$ while the worst performance for all is only $-3.27 \%$ at threshold $0.05 \%$. Both in two currencies, strategy 2 shows a remarkable correction in returns. From the view of balance drawdown in table $\mathrm{V}$ and table VI, strategy 2 does not show stable correction. There are both 7 thresholds drawdown showing a drop for the two currencies but the other 3 has different degrees of rise. The profit factor has also the same correction as the returns do. They have better figures than those in strategy 1 . The only exception is at threshold $0.05 \%$ for GBP/USD. Strategy 2 performs worse than strategy 1 which $-0.33 \%$ against $0.77 \%$. The total trades in strategy 2 is almost identical to the strategy1's because they run similar algorithm. In winning rate, there are 8 out of 10 instances for EUR/USD getting higher in strategy 2 but this number is only 4 out of 10 for GBP/USD.

TABLE V. MAX BALANCE DRAWDOWN OF EUR/USD

\begin{tabular}{|l|l|l|l|l|}
\hline Threshold & Strategy 1 & Strategy 2 & Strategy 3 & Strategy 4 \\
\hline $0.01 \%$ & $32.49 \%$ & $29.57 \%$ & $4.43 \%$ & $4.40 \%$ \\
\hline $0.03 \%$ & $42.91 \%$ & $50.47 \%$ & $2.72 \%$ & $1.86 \%$ \\
\hline
\end{tabular}

\begin{tabular}{|l|l|l|l|l|}
\hline $0.05 \%$ & $17.74 \%$ & $22.36 \%$ & $3.45 \%$ & $1.53 \%$ \\
\hline $0.07 \%$ & $28.61 \%$ & $9.93 \%$ & $3.35 \%$ & $1.93 \%$ \\
\hline $0.09 \%$ & $11.34 \%$ & $5.53 \%$ & $2.97 \%$ & $1.79 \%$ \\
\hline $0.1 \%$ & $13.64 \%$ & $18.87 \%$ & $3.91 \%$ & $2.68 \%$ \\
\hline $0.3 \%$ & $1.53 \%$ & $1.30 \%$ & $2.25 \%$ & $0.55 \%$ \\
\hline $0.5 \%$ & $2.59 \%$ & $1.99 \%$ & $2.86 \%$ & $0.00 \%$ \\
\hline $0.7 \%$ & $2.33 \%$ & $2.97 \%$ & $3.77 \%$ & $0.00 \%$ \\
\hline $0.9 \%$ & $3.13 \%$ & $0.86 \%$ & $3.39 \%$ & $0.00 \%$ \\
\hline
\end{tabular}

TABLE VI. MAX BALANCE DRAWDOWN OF GBP/USD

\begin{tabular}{|l|l|l|l|l|}
\hline Threshold & \multicolumn{1}{|c|}{ Strategy 1 } & \multicolumn{1}{|c|}{ Strategy 2 } & \multicolumn{1}{|c|}{ Strategy 3 } & Strategy 4 \\
\hline $0.01 \%$ & $99.95 \%$ & $30.89 \%$ & $19.49 \%$ & $2.88 \%$ \\
\hline $0.03 \%$ & $56.21 \%$ & $24.18 \%$ & $16.05 \%$ & $4.84 \%$ \\
\hline $0.05 \%$ & $20.74 \%$ & $22.76 \%$ & $17.13 \%$ & $4.73 \%$ \\
\hline $0.07 \%$ & $36.32 \%$ & $7.82 \%$ & $17.99 \%$ & $4.86 \%$ \\
\hline $0.09 \%$ & $15.06 \%$ & $5.40 \%$ & $18.39 \%$ & $5.70 \%$ \\
\hline $0.1 \%$ & $9.11 \%$ & $35.48 \%$ & $15.73 \%$ & $4.35 \%$ \\
\hline $0.3 \%$ & $6.02 \%$ & $4.35 \%$ & $8.63 \%$ & $0.83 \%$ \\
\hline $0.5 \%$ & $3.83 \%$ & $4.55 \%$ & $16.93 \%$ & $1.19 \%$ \\
\hline $0.7 \%$ & $1.62 \%$ & $1.46 \%$ & $13.21 \%$ & $0.00 \%$ \\
\hline $0.9 \%$ & $1.00 \%$ & $0.35 \%$ & $17.90 \%$ & $2.05 \%$ \\
\hline
\end{tabular}

Compare to the strategy 1 , strategy 2 edits the exit position of order using trailing stop instead of fixed stop loss level. It does produce better performance and remarkable correction in most cases. Trailing stop is considerable tool to improve a directional change event based strategy. It fits directional 
change event very well because it catches the potential profits in the directional change overshoot period without risking much.

Strategy 3 use another idea to close a trade rather than using stop loss and take profit. It has the same position entrance point asthe confirmation point is. To close the order, strategy 3 use a close threshold which is related to the overshoot value in each directional change event. By defining the close threshold formula, strategy 3 can automatically trade. $80 \%$ of the test in EUR/USD generates positive returns while the worst two has only $-0.30 \%$ and $-0.83 \%$ return. The highest return occurs at threshold $0.03 \%$ where it is $21.32 \%$. There are Averagely it has $9.42 \%$ return in EUR/USD. From table IV, there are half instances succeed in making profits where the highest is $12.48 \%$ at threshold $0.03 \%$ and the lowest is $15.00 \%$ at threshold $0.9 \%$. The range of returns is narrow than strategy 1 and the volatility of returns is also lower than strategy 1. In balance drawdown table V and VI, EUR/USD holds all under 5\% which is excellent performance, and GBP/USD has all under $20 \%$. There are 7 profit factors are higher than strategy 1 in both currency pair. The highest profit factor reaches 1.55 at threshold $0.9 \%$ while the worst one is only 0.93 at threshold at threshold $0.7 \%$ for EUR/USD. This number is 1.13 at threshold $0.3 \%$ and 0.57 at threshold $0.9 \%$ for GBP/USD. Total trades decrease when threshold increases. GBP/USD has more trades than EUR/USD. For each threshold, strategy 3 has more trades than strategy 1 has. The average winning rate for both currencies is about $55 \%$ and the volatility of all the rates is low. Most of the winning rate gathers around average level. In all, strategy 3 has good performance in EUR/USD though not better than strategy 1. However, in GBP/USD it has better returns than strategy 1 normally has. The advantage is strategy 3 has a little more frequencies and the drawdown is very low and stable. The wining rate of strategy 3 is also stable. also another 4 thresholds test having returns above $10 \%$.

\begin{tabular}{|l|l|l|l|l|}
\hline Threshold & Strategy 1 & \multicolumn{1}{|c|}{ Strategy 2 } & Strategy 3 & Strategy 4 \\
\hline $0.01 \%$ & 0.81 & 1.05 & 0.99 & 1.05 \\
\hline $0.03 \%$ & 0.92 & 1.02 & 1.04 & 1.02 \\
\hline $0.05 \%$ & 0.92 & 0.99 & 1.01 & 1.07 \\
\hline $0.07 \%$ & 0.91 & 1.05 & 1.02 & 1.01 \\
\hline $0.09 \%$ & 0.92 & 1.02 & 0.98 & 0.89 \\
\hline $0.1 \%$ & 0.96 & 1.10 & 1.01 & 0.98 \\
\hline $0.3 \%$ & 1.06 & 1.15 & 1.13 & 2.43 \\
\hline $0.5 \%$ & 1.04 & 0.99 & 0.72 & 1.62 \\
\hline $0.7 \%$ & 1.07 & 1.18 & 0.74 & - \\
\hline $0.9 \%$ & 0.82 & 1.74 & 0.57 & 0 \\
\hline Threshold & Strategy 1 & Strategy 2 & Strategy 3 & Strategy 4 \\
\hline $0.01 \%$ & 20,250 & 20,252 & 22,981 & 5,877 \\
\hline $0.03 \%$ & 7,614 & 7,619 & 9,296 & 2,017 \\
\hline $0.05 \%$ & 3,849 & 3,897 & 4,956 & 853 \\
\hline $0.07 \%$ & 2,373 & 2,377 & 3,066 & 420 \\
\hline $0.09 \%$ & 1,629 & 1,627 & 2,106 & 227 \\
\hline $0.1 \%$ & 1,329 & 1,336 & 1,749 & 169 \\
\hline $0.3 \%$ & 207 & 207 & 281 & 6 \\
\hline $0.5 \%$ & 71 & 71 & 192 & 1 \\
\hline $0.7 \%$ & 40 & 40 & 59 & 1 \\
\hline $0.9 \%$ & 31 & 31 & 40 & 0 \\
\hline
\end{tabular}

TABLE X. TOTAL TRADES OF GBP/USD

\begin{tabular}{|l|l|l|l|l|}
\hline Threshold & Strategy 1 & \multicolumn{1}{|c|}{ Strategy 2 } & \multicolumn{1}{|c|}{ Strategy 3 } & Strategy 4 \\
\hline $0.01 \%$ & 6,104 & 21,918 & 24,316 & 6,319 \\
\hline $0.03 \%$ & 9,047 & 8,914 & 10,822 & 2,422 \\
\hline $0.05 \%$ & 4,850 & 4,872 & 6,063 & 1,183 \\
\hline $0.07 \%$ & 3,093 & 3,060 & 3,915 & 594 \\
\hline $0.09 \%$ & 2,137 & 2,137 & 2,748 & 327 \\
\hline $0.1 \%$ & 1,793 & 1,826 & 2,355 & 245 \\
\hline $0.3 \%$ & 312 & 304 & 431 & 20 \\
\hline $0.5 \%$ & 123 & 125 & 170 & 11 \\
\hline $0.7 \%$ & 73 & 73 & 96 & 1 \\
\hline $0.9 \%$ & 43 & 22 & 60 & 1 \\
\hline
\end{tabular}

TABLE VII. PROFIT FACTOR OF EUR/USD

\begin{tabular}{|l|l|l|l|l|}
\hline Threshold & Strategy 1 & Strategy 2 & Strategy 3 & Strategy 4 \\
\hline $0.01 \%$ & 0.96 & 0.98 & 1.04 & 0.96 \\
\hline $0.03 \%$ & 1.08 & 1.14 & 1.13 & 1.00 \\
\hline $0.05 \%$ & 1.11 & 1.12 & 1.15 & 1.13 \\
\hline $0.07 \%$ & 1.19 & 1.38 & 1.15 & 1.08 \\
\hline $0.09 \%$ & 1.22 & 1.33 & 1.18 & 0.95 \\
\hline $0.1 \%$ & 1.24 & 1.24 & 1.08 & 0.80 \\
\hline $0.3 \%$ & 1.07 & 1.21 & 1.32 & 0.54 \\
\hline $0.5 \%$ & 0.97 & 0.98 & 0.98 & - \\
\hline $0.7 \%$ & 0.65 & 0.8 & 0.93 & - \\
\hline $0.9 \%$ & 1.17 & $1.91 \quad$ & 1.55 & - \\
\hline
\end{tabular}

TABLE VIII. PROFIT FACTOR OF GBP/USD

Profit factors are not applicable at some thresholds.

TABLE IX. TOTAL TRADES OF EUR/USD
TABLE XI. WINNING RATE OF EUR/USD

\begin{tabular}{|l|l|l|l|l|}
\hline Threshold & \multicolumn{1}{|c|}{ Strategy 1 } & \multicolumn{1}{|c|}{ Strategy 2 } & \multicolumn{1}{|c|}{ Strategy 3 } & Strategy 4 \\
\hline $0.01 \%$ & $32.50 \%$ & $38.54 \%$ & $59.04 \%$ & $56.13 \%$ \\
\hline $0.03 \%$ & $71.60 \%$ & $68.74 \%$ & $57.11 \%$ & $54.88 \%$ \\
\hline $0.05 \%$ & $68.95 \%$ & $72.29 \%$ & $57.12 \%$ & $58.62 \%$ \\
\hline $0.07 \%$ & $32.36 \%$ & $79.94 \%$ & $56.75 \%$ & $55.48 \%$ \\
\hline $0.09 \%$ & $19.58 \%$ & $70.44 \%$ & $56.84 \%$ & $55.07 \%$ \\
\hline $0.1 \%$ & $62.60 \%$ & $67.29 \%$ & $55.12 \%$ & $50.89 \%$ \\
\hline $0.3 \%$ & $76.33 \%$ & $74.88 \%$ & $54.80 \%$ & $33.33 \%$ \\
\hline $0.5 \%$ & $59.15 \%$ & $49.30 \%$ & $50.00 \%$ & $100 \%$ \\
\hline $0.7 \%$ & $50.00 \%$ & $60.00 \%$ & $50.85 \%$ & $100 \%$ \\
\hline $0.9 \%$ & $48.39 \%$ & $80.65 \%$ & $57.50 \%$ & - \\
\hline
\end{tabular}


TABLE XII. WINNING RATE OF GBP/USD

\begin{tabular}{|l|l|l|l|l|}
\hline Threshold & Strategy 1 & \multicolumn{1}{|c|}{ Strategy 2 } & Strategy 3 & Strategy 4 \\
\hline $0.01 \%$ & $70.93 \%$ & $52.79 \%$ & $60.07 \%$ & $58.95 \%$ \\
\hline $0.03 \%$ & $69.72 \%$ & $65.90 \%$ & $58.04 \%$ & $55.24 \%$ \\
\hline $0.05 \%$ & $89.18 \%$ & $71.51 \%$ & $56.29 \%$ & $54.86 \%$ \\
\hline $0.07 \%$ & $89.07 \%$ & $75.03 \%$ & $56.60 \%$ & $55.05 \%$ \\
\hline $0.09 \%$ & $69.54 \%$ & $75.29 \%$ & $56.00 \%$ & $54.74 \%$ \\
\hline $0.1 \%$ & $65.64 \%$ & $47.32 \%$ & $55.88 \%$ & $57.55 \%$ \\
\hline $0.3 \%$ & $70.51 \%$ & $71.71 \%$ & $57.77 \%$ & $65.00 \%$ \\
\hline $0.5 \%$ & $67.48 \%$ & $62.40 \%$ & $51.76 \%$ & $54.55 \%$ \\
\hline $0.7 \%$ & $56.16 \%$ & $56.16 \%$ & $56.25 \%$ & $100 \%$ \\
\hline $0.9 \%$ & $76.74 \%$ & $77.27 \%$ & $41.67 \%$ & $0 \%$ \\
\hline
\end{tabular}

Strategy 4 uses the similar algorithm as strategy 3 uses. We combine a technical analysis tool - DMI to test the algorithm. We only run the strategy when DMI shows there is a significant trend in the market.

In the last test at threshold $0.9 \%$ there is no transaction in EUR/USD. There are four out of ten having profits in EUR/USD, where in each threshold it is commonly worse than the performance in strategy 3. In GBP/USD, the result is 7 out of 10 , ranging from $-2.27 \%$ to $5.88 \%$, which is not a significant profitable strategy. The maximum drawdown in strategy 4 varies under $5 \%$, which is always lower than individual test in strategy 3. Profit factor does not have clear relationship compared to strategy 3 . The total trades reduces a lot, almost one fourth of the number in strategy 3 while the winning rate averagely equals to the strategy 3's. The modification of strategy 4 does not improve the probability. In some cases, it is even worse. The winning rate does not show improvement neither. The only correction is the balance drawdown. Over all, the DMI is not a good tool to run in the strategy with directional change. The reason may be that as a technical analysis indicator, DMI is made of a set of previous prices. The trend it tells is always delayed some periods when the market trend moves.

\section{CONCLUSIONS}

The majority of trading models provide trading rules based on time series. Few trading models are developed under the DC framework. In this paper, we introduce four trading strategies using the DC concept. We also explore combining DC concepts with traditional technically analysis tools. We run the strategies using two most popular currency pairs, EUR/USD and GBP/USD. The data are divided into a back testing period and a demo testing period. The back testing is used forparameter optimization and the demo testing period is used to simulate the real market run. We measure the total profits, profit factor, max drawdown, total trades, and winning rates of the results in demo testing.Trading frequency is implemented by using different thresholds. Evidence shows that strategy $1 \& 3$ generated profits in many thresholds, and that trailing stop is a good correction tool to strategy 1 . The evidence also shows that DMI technical analysis may not be suitable for DC strategies. We verify the performance of the sustainable scaling laws in FX data by building scale invariant trading strategies and their profitability.

\section{REFERENCES}

[1] J. Vanstone, T. Hahn and G. Finnie, "Developing high-frequency foreign exchange trading systems," in the proceeding of 25 th Australasian Finance and Banking Conference, Sydney, Australia, 2012. SSRN: http://ssrn.com/abstract=2132390.

[2] Mitchell Ratner, Ricardo P.C. Leal, "Tests of technical trading strategies in the emerging equity markets of Latin America and Asia," Journal of Banking \& Finance, vol. 23, pp. 1887-1905, Dec. 1999

[3] Fei Wang, Philip L.H. Yu, David W. Cheung, "Combining technical trading rules using particle swarm optimization," Expert Systems with Applications, vol.41, pp 3016-3026, May 2014.

[4] Chabot, Benjamin, Eric Ghysels, and Ravi Jagannathan Momentum trading, return chasing, and predictable crashes. No. w20660. National Bureau of Economic Research, 2014.

[5] Hong, K. J., and Stephen Satchell. "Time series momentum trading strategy and autocorrelation amplification." Quantitative Finance 15.9 (2015): 1471-1487.

[6] J.-S. Chen, J.-L. Hou, S.M. Wua and Y.W. Chang-Chien, "Constructing investment strategy portfolios by combination genetic algorithms," Expert System With Application, vol. 36, pp. 3824-3828, 2009.

[7] Guillaume, M. Dacorogna, R. Davé, U. Müller, R. Olsen and O. Pictet, "From the bird's eye to the microscope: A survey of new stylized facts of the intra-daily foreign exchange markets," Finance and Stochastic, vol. 1, no. 2, pp. 95-129, 1997.

[8] J. Glattfelder, A. Dupuis and R. Olsen, "Patterns in high-frequency FX data: Discovery of 12 empirical scaling laws," Quantitative Finance, vol. 11, no. 4, pp. 599-614, 2011.

[9] T. Bisig, A. Duouis, and V. Impagliazzo, "The scale of market quakes”, Quantitative Finance, vol. 12, no. 4, pp. 501-508, 2012.

[10] P. K. Tsang, R. Tao, A. Serguieva and S. Ma, "Profiling highfrequency equity price movements in directional changes," Quantitative Finance, vol. 17, no. 2, pp. 217-225, 2017.doi:10.1080/14697688.2016.1164887

[11] A. Dupuis and R. Olsen, "High frequency finance: using scaling laws to build trading models," in Handbook of exchange rates, Ed.: J. James et al, Wiley, 2012, pp. 563-582.

[12] J. Gypteau, F. Otero and M. Kampouridis, "Directional changes based trading strategy with genetic programming," in: A. M Mora, and G. Squillero, Ed., EvoApplications, EvoStar. Springer, 2015, pp. 1-12.

[13] Bakhach, Amer, et al. "Backlash Agent: A trading strategy based on Directional Change." Computational Intelligence (SSCI), 2016 IEEE Symposium Series on. IEEE, 2016.

[14] G.B. West, J.H. Brown, and B.J. Enquist. Ageneral model for the orgin of allometric scaling laws in biology. Science, 276(5309):122-126, 1997.

[15] U. A. M“uller, M. M. Dacorogna, R. B. Olsen, O. V. Pictet, M. Schwarz, and C. Morgenegg. Statistical study of foreign exchange rates, empirical evidence of a price change scaling law, and intraday analysis. J. Bank. Financ., 14:1189-1208, 1990.

[16] R. Pardo, "The Evaluation and optimization of trading strategies," 2nd edition, NJ, USA: John Wiley \& Sons, 2008. 